\section{Latent Variable}

Matthew J. L. Page

Psychology, Allegheny Health Network,

Pittsburgh, PA, USA

\section{Definition}

A latent variable represents a construct that is unobserved and not directly measurable. Latent variables are often hypothetical or abstract concepts. In the behavioral sciences, examples of such variables might include quality of life, depression, and selective attention. In contrast, measures such as a single neuropsychological subtest score or a behavioral rating scale score are observed variables. Such a measure can also be referred to as an indicator variable, as it indicates or reflects the latent construct. Whereas a single indicator variable measures one dimension of behavior, the latent variable can collectively explain a wider range of behaviors or processes.

Some statistical analyses, such as structural equation modeling (SEM), use latent variables as a representation of the shared variance (or covariance) of multiple indicator variables. Although observed measures inevitably entail measurement error, latent variables account for this error and provide a clearer representation of the construct. In factor analysis, underlying latent factors are determined by identifying how individual items or subtests are correlated with each other. In path model diagrams, indicator variables are typically depicted using rectangles and latent variables using circles or ovals.

\section{Current Knowledge}

A classic example of a latent variable in the cognitive sciences is Charles Spearman's $g$ factor, reflecting general intelligence. Although intelligence cannot be directly measured, performance across a series of standardized intelligence tests is thought to collectively reflect the underlying general intelligence factor, $g$. Even though the individual tasks may be very different, the commonality across their performance scores theoretically represents the global intelligence factor. Subsequent theorists and researchers have proposed sub-factors to general intelligence, such as working memory and fluid reasoning, which are also latent variables.

\section{See Also}

- Factor Analysis

- Structural Equation Modeling

\section{Further Readings}

Spearman, C. (1904). "General intelligence," objectively determined and measures. The American Journal of Psychology, 15(2), 201-292. doi:10.2307/1412107. 